\title{
HELAS IT-platform: A new tool for the European Helio- and Asteroseismology community.
}

\author{
S. J. Jiménez-Reyes ${ }^{1}$, J. Quintero Nerkhorn ${ }^{1}$, P. L. Pallé ${ }^{1}$, and HELAS Board ${ }^{2}$ \\ ${ }^{1}$ Instituto de Astrofísica de Canarias, c/Va Lactea, \\ 38205 La Laguna, Tenerife, Spain \\ 2 Helas Project Office, Kiepenheuer-Institut für Sonnenphysik, \\ Schneckstr. 6, D-79104 Freiburg, Germany
}

\begin{abstract}
HELAS is a Coordination Action funded under the European Commission's Sixth Framework Programme (FP6) since April 1st, 2006 till March 31st, 2010. The HELAS Consortium includes 10 partner institutions that host researchers active in helio- and asteroseismology. This initiative offers a unique chance to advance the field further by coordinating the activities of researchers, enhancing the quality and quantity of science performed in Europe. The HELAS Forum is one of its Networking Activities. Its main goal is to encourage, provide, and support the tools, activities, and initiatives emanating from the European helio- and asterosesimology scientific communities. Thus, the HELAS Forum should ensure European competence and competitiveness in these areas by better coordinating the groups. It also enhances the synergies between the helioand astero communities and between those and other non-European related activities. Here, we present the Helas Forum IT-platform (www.helas-eu.org), developed to facilitate the coordinated activities such as data and software package exchange, information about workshops and meetings, knowledge of the community, etc., as well as to allow tracking the development of HELAS activities.
\end{abstract}

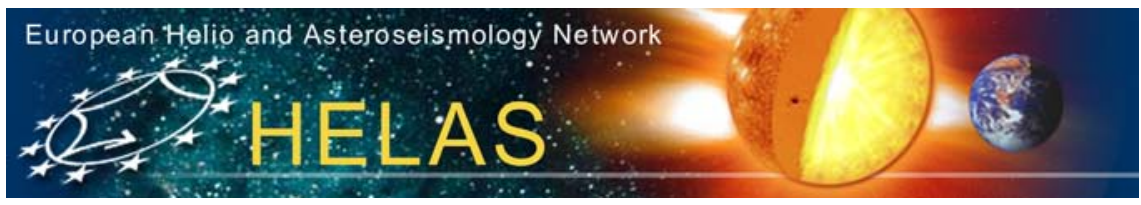

Figure 1: Header of the HELAS IT website 


\section{Introduction}

Over the last 25 years, helioseismology has tremendously increased our knowledge about the physical properties of the Sun. Precise measurements of the oscillations on the solar surface are carried out with worldwide networks of observing stations as well as dedicated space experiments. These seismic investigations of the Sun are the only possibility to derive information about the solar interior: its dynamical and structural properties. Asteroseismology continues this success by investigating stellar interiors.

In the coming years, various innovative experiments will start delivering data that would allow exciting scientific results about solar and stellar interiors. An unheard volume of data will be collected by new space experiments and high-tech ground-based observatories. European science requires the best intellectual integration to efficiently structure the investigations that make use of these data. The objective of the Helioand Asteroseismology Network HELAS is to co-ordinate activities among those European institutions and organizations that support Europe's major research groups in the field and further to progressively incorporate the incipient ones (see Roth et al. 2006). These groups are widely spread across Europe. The transfer of knowledge, data, and data analysis techniques through HELAS will structure of this field of research to prepare the European research community for important opportunities in the immediate future. These opportunities include the CNES missions COROT (Convection, Rotation \& planetary Transits) launched in December 2006, the NASA space missions Solar Dynamics Observatory (SDO) and KEPLER, and the CNES mission PICARD, all of which will become operational within the next three years, and the ESA mission Solar Orbiter in the next decade.

Combining the core competences of the individual research groups through the network activities of HELAS, the proposed activity will ensure European competence and competitiveness in this research area by spreading expertise, increasing the volume and quality of the European scientific output in the field.

The HELAS Networking Activities are conceived to coordinate activities in helioand asteroseismology. Three of them are designed to support and prepare the science performed with existing and upcoming facilities: Global Helioseismology, Local Helioseismology, and Asteroseismology. In particular thanks to the space missions (e.g., MOST, COROT), others under development (e.g., Kepler) and the possible extension of the ground-based observations (e.g., SONG) Asteroseismology (the less developed branch) stands clearly on the threshold of a large increase not only in the number of stars for which data are available, but also in the lengths of those data sets. The detection of oscillations in a large number of stars offers the prospect of being able to test the actual models of stellar evolution.

Another networking activity is Public Outreach, aiming the discussion and dissemination of science and knowledge at the general public. Last, the Forum should ensure and facilitate coordinated activities such as data and software package exchange, information about workshops and meetings, knowledge, etc., as well as to allow tracking the development of the HELAS activities. The proposed objectives will be reached thanks to the HELAS IT platform (www.helas-eu.org), which was created with the intention of becoming the reference for the field. 


\section{The HELAS IT platform}

The purpose of this initiative is to create a unique reference website for the helioand asteroseismology European community, to be a unifying force element and a common meeting-place for the community. This website will be a platform (HELAS IT-platform) of information and scientific products. Moreover, it will serve as an adequate forum for the scientific community. The users (and beneficiaries) of the services and facilities will be:

- European and worldwide astronomers actively working or interested in the fields of helio- and asteroseismology

- Under- and postgraduate students in scientific domains (astrophysics, physics, etc.)

- Elementary scholarships and the general public.

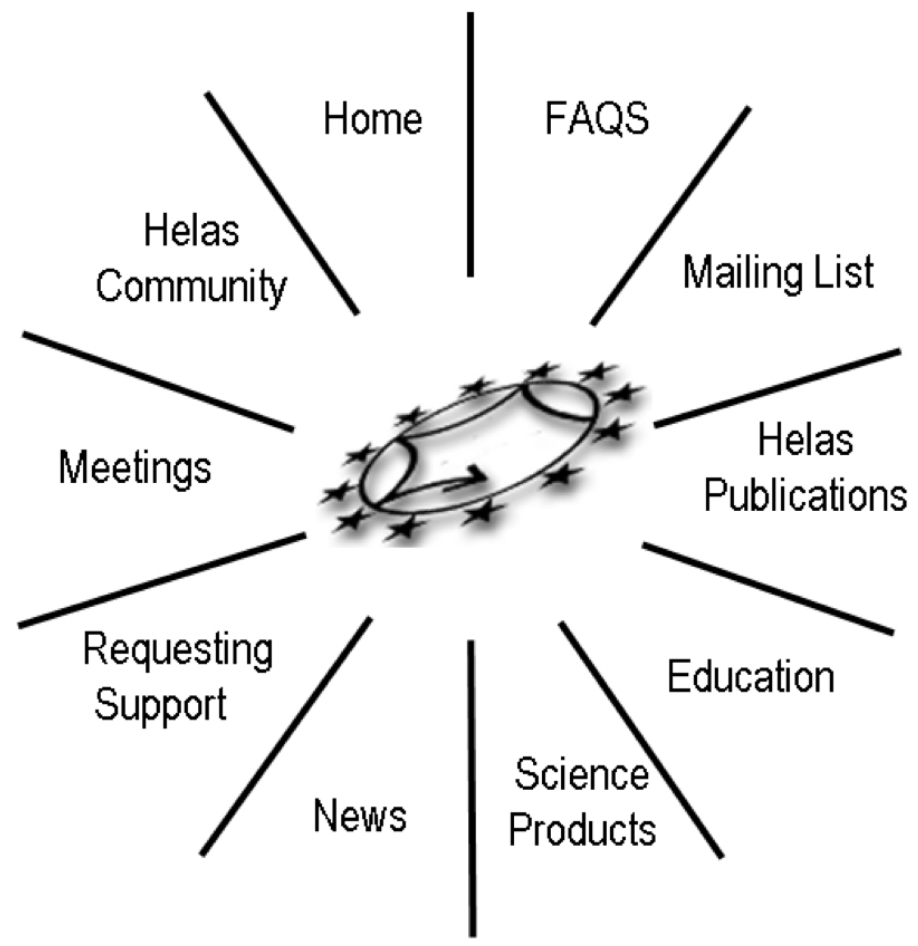

Figure 2: Structure of the main body 
To fully accomplish the proposed objectives for the HELAS IT-platform, the users must easily manage given aspects of the website. Thus, via a simple browser-based interface, the users will be able to easily post news, job announcements, and press releases, or download software or the proceedings of the last meeting, etc. Thus, it was decided to use a content management system based on Joomla, since it is especially suitable for our purposes. Further, Joomla is extremely extensible, allowing for instance RSS feeds, forums, calendars, etc.

With traditional website designs, there is a bottleneck whereby the webmaster must enter all the content into the site. In a large organization like the HELAS Community, it is possible to have designated contributors each with web editing software, but the costs add up, and each person must be trained to work with HTML. The larger the group of contributors, the higher the cost.

\section{HELAS Board \\ Coordinator: Oskar von der Lühe Project Scientist: Markus Roth Project Office, Network Coordination Team}

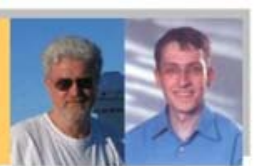

NA2: HELAS Forum

Pere L. Pallé

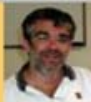

NA3: Global Helioseismology

Michael Thompson

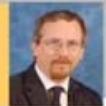

NA4: Local Helioseismology

Laurent Gizon

NA5: Asteroseismology

Conny Aerts

NA6: Public Outreach

Jørgen Christensen-Dalsgaard

Figure 3: Network Activities and responsibles.

Within Joomla, the developer sets up a template, and the administrator can give permission to others who would simply need internet access to log in and enter content on their own schedule. There are tools built in to the back end to customize the look and feel of the page, much like the tools you would use for creating a Word document. The administrator can allow others to publish directly, or have it approved first. Four types of users are defined, each with different privileges. 
- Level 0: Full administrative control of the site ('root' level). This user level will, in particular, moderate the requests received by the community members to include new material in the platform (job offers information, recent papers publications, meetings information, etc.).

- Level 1: Members of the Helas Board and of the Helas Project Office (with access to the Project Management Material).

- Level 2: Registered members, able to download and submit material. This will be the most common level as it refers to the access for scientists and students to the products and information provided by the HELAS IT-platform.

- Level 3: Guests, who use the website without login.

To register on the HELAS IT-platform, the user should fill in the 'Login Form' that appears on the left table of the main page. Once the support technician approves the new member, he/she can optionally join 'Who is who'. In this section, the new user can indicate his or her expertise, personal website, affiliation, address, etc. Note that registered users can always change their profile using the options that appear in 'User Menu' (botton-left panel).

\section{Facilities}

The main page of the IT platform contains the standard elements that give the user a global overview. The basic elements are the top area (titles and logos), a central welcome area, a left side tabs index, and another right side table with the 'Latest News' in different topics. Last, the 'User Registration Area' appears on the bottom on the right table. The flowchart in Figure 2 shows a general view of the main options of the left table. Here we describe the most important.

- Home. Description of the HELAS Co-ordination Action and the corresponding established Consortium: management bodies, network activities (NAs) description, main objectives, as well as existing opportunities to participate and become involved in their objectives.

- HELAS Community. This contains effective interactive tools to obtain quick and detailed knowledge of the HELAS Community in general with additional information about members' current positions, affiliations, fields of expertise and interest, associated research projects, etc. The database mainly contains activities and people in European institutions, but there are plans to widen the scope to non-European institutions.

- Meetings. This provides detailed information on conferences and workshops related to the field. The events are classified (and colour coded for the visual planning tool) in different categories depending of the main topic and if they are sponsored by HELAS. The interactive calendar allows a quick look at the temporal planning of the events at scales of various years.

- Requesting support. The user will find information on the different options to get support as well as the main guidelines to apply for it (see also next section). 


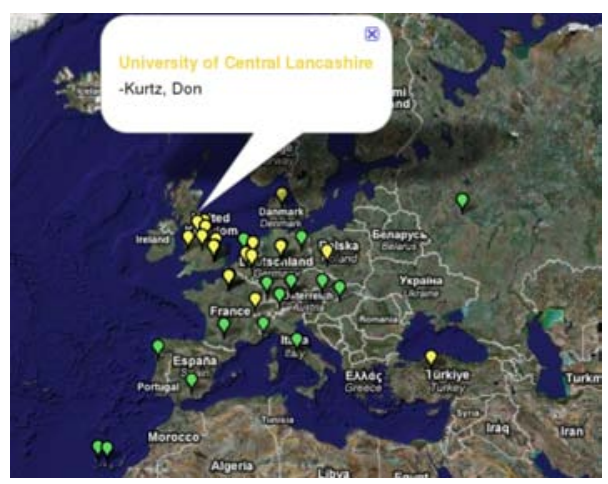

Figure 4: The figure shows the interactive map, where any user can find information about institutions and registered users not only in Europe, but also around the world.

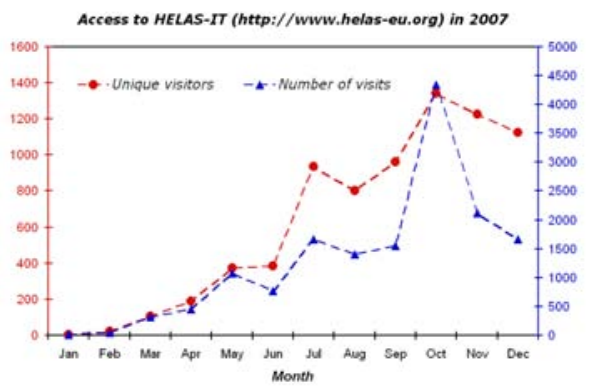

Figure 5: The figure shows the unique number of visits. As it can be seen, it has increased gradually. There are already 109 registered users so far, which we hope will increase.

- News. Archive of different types of news (job opportunities, latest papers, meetings and workshops, press release, etc.). The most recent ones appear on the right tab of the website. This key area nourishes itself not only from the Networking Activities chairs but also from any registered scientists. Thus, registering implies the open possibility to submit any kind of news to be included in the HELAS IT-Platform, always under the supervision of the moderator. This section also contains the previous NewsLetter, sent monthly to all registered users and containing the news received during the month.

- Science Products. As one of the HELAS objectives is precisely the dissemination and public distribution of ad-hoc scientific products, this area is relevant. Specific HELAS products or links to their location can be found here. Scien- 


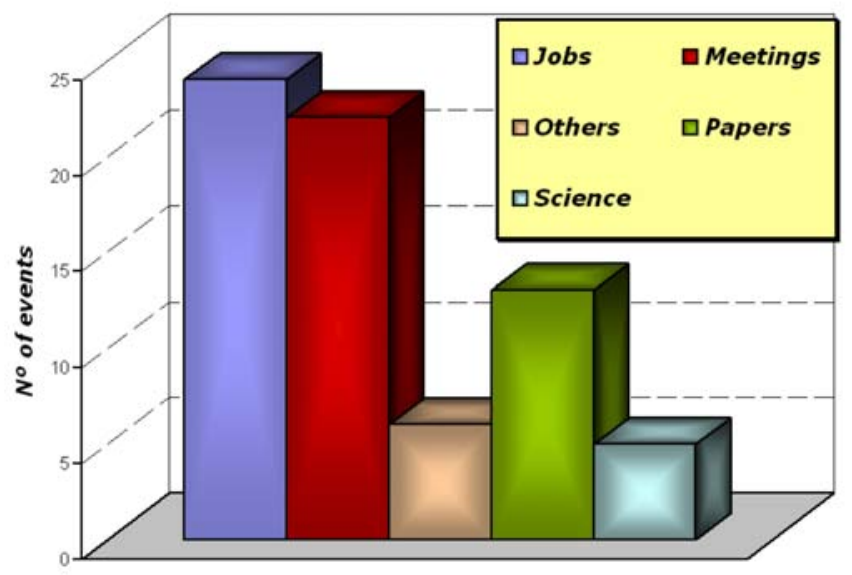

Figure 6: Number of news classified in different catergories announced so far.

tific products consist of software packages, analysis tools, stellar-solar model codes, basic helio- and asteroseismology data, etc. An ad-hoc ftp site exists to facilitate the uploading or downloading, with the adequate safety mechanisms.

- Education. This contains written material addressed to under- and postgraduate students in astrophysics or related fields. This section is split right now into two sections: multimedia material and lectures notes.

- Publications. This contains an archive of the papers sponsored by HELAS and all the proceedings of the HELAS workshop and conferences.

- Forum. This provides a place for general discussions on scientific topics related to the field. The most recent contributions will appear below the 'Latest News' in the right table.

- Related Projects. All the organized activities related to helio- and asteroseismology are posted, updated, and classified in different sections.

- Mailing list. This tool allows the sending of messages that may have significant impact on the HELAS community and therefore appropriate for mass distribution. The messages must be approved by the HELAS Project Manager. 


\section{Request of Support}

One of the main objectives of the HELAS Forum is to stimulate, support, and enhance the existing and new initiatives emanating from the helio- and asteroseismology community, whose aims are in accordance with those of the HELAS Coordination Action, described in the various Network Activities: Forum, Global Helioseismology, Local Helioseismology, Asteroseismology and Public Outreach.

The Forum Network Activity can support scientists to:

1. Participate in already planned HELAS workshops or organize specific workshops and meetings, if they are relevant tothe HELAS goals

2. Generate and exploit synergies between HELAS activities and other networks

3. Invite scientists belonging to the HELAS Community to a European institution related to the field

The budget for those activities is limited and the principle of equal opportunities based on the quality and scientific interest isof major importance in allocating the resources. The user will find in the IT-platform the guidelines on how the community can apply for this limited support.

Note also that other HELAS Network Activities have a given budget for invitations and synergies. So, if a user is interested in working or collaborating with a participant in those activities, he/she should contact the corresponding coordinator of that Network Activity.

Acknowledgments. This work was supported by the European Helio- and Asteroseismology Network (HELAS), a major international collaboration funded by the European Commission's Sixth Framework Programme.

\section{References}

Roth, M., et al. 2006, ESA-SP 624, 130 
